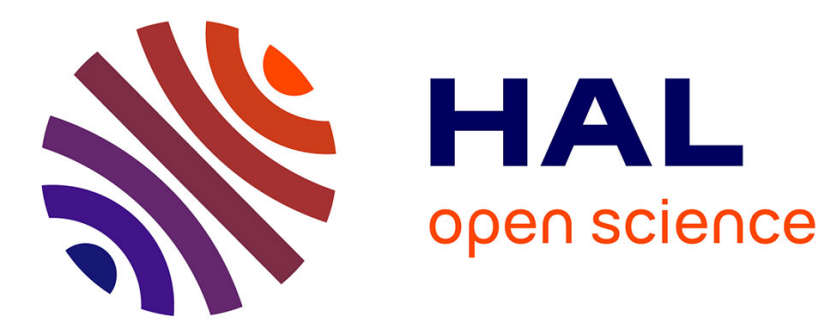

\title{
Tomography in standing trees: revisiting the determination of acoustic wave velocity
} Andrés Arciniegas, Loïc Brancheriau, Philippe Lasaygues

\section{To cite this version:}

Andrés Arciniegas, Loïc Brancheriau, Philippe Lasaygues. Tomography in standing trees: revisiting the determination of acoustic wave velocity. Annals of Forest Science, 2015, 72 (6), pp.685-691. 10.1007/s13595-014-0416-y . hal-01284203

\section{HAL Id: hal-01284203 https://hal.science/hal-01284203}

Submitted on 7 Mar 2016

HAL is a multi-disciplinary open access archive for the deposit and dissemination of scientific research documents, whether they are published or not. The documents may come from teaching and research institutions in France or abroad, or from public or private research centers.
L'archive ouverte pluridisciplinaire HAL, est destinée au dépôt et à la diffusion de documents scientifiques de niveau recherche, publiés ou non, émanant des établissements d'enseignement et de recherche français ou étrangers, des laboratoires publics ou privés. 


\title{
Tomography in standing trees: revisiting the determination of acoustic wave velocity
}

\author{
Andrés Arciniegas • Loïc Brancheriau • \\ Philippe Lasaygues
}

Received: 26 March 2014 / Accepted: 28 July 2014 / Published online: 13 August 2014

(C) INRA and Springer-Verlag France 2014

\begin{abstract}
- Context The quality of acoustic tomographic images in standing trees is mainly function of the accuracy of the acoustic velocity computation. Improving the acoustic velocity determination is, furthermore, of great interest because acoustic tools are widely used in nondestructive testing of wood.

- Aims Four different signal processing algorithms were used (1) to study the effect of the signal dynamic on the velocity determination, (2) to determine the validity range of each computation method, and (3) to compare the behavior between a homogeneous material and wood.

- Methods The experiments were performed using the conventional experimental protocol for the ultrasonic characterization of materials in a tank (normal incidence transmission at $500 \mathrm{kHz}$ ). A polyurethane resin (homogeneous material) and two wood species (Bagassa guianensis Aubl., Milicia excelsa (Welw.) C.C. Berg) were used for the experiments.

- Results Computed velocity increased as the noise level increased. The Hinkley method appeared to be the most exact when the noise level exceeded $10 \mathrm{~dB}$. The Fisher method was that most suitable for very noisy signals. No difference was found between the resin and the wood samples.
\end{abstract}

\section{Handling Editor: Jean-Michel Leban}

Contribution of the co-authors Andrés Arciniegas: performing the experimental tests, running the data analysis, and writing the manuscript. Loïc Brancheriau: contribution to the analysis and discussion, and writing the manuscript.

Philippe Lasaygues: supervising the experimental tests, and contribution to the analysis and discussion.

A. Arciniegas $\cdot$ P. Lasaygues

CNRS, LMA UPR 7051, Marseille, France

L. Brancheriau $(\bowtie)$

CIRAD, UR BioWooEB - UMR AMAP, Montpellier, France

e-mail: loic.brancheriau@cirad.fr
- Conclusion A combination of the Fisher and Hinkley methods in the same algorithm would yield the most accurate acoustic velocity determinations in the tomography of standing trees.

Key message Wood acoustic velocity determination is affected by the wavelength and the detection algorithm used. The Fisher algorithm is optimal with high signal attenuation; otherwise, the Hinkley algorithm should be used.

Keywords Wave velocity . Ultrasonic testing · Tomography

\section{Introduction}

Wood is an inhomogeneous material with anisotropic properties and a high degree of variability. Studying wood quality and intraspecific variability yields information that is useful for clonal selection, classification of wood material, and tree risk management in urban areas. Nondestructive testing (NDT) methods are used to assess the quality of wood (Pellerin and Ross 2002; Bucur 2003) and include acoustic and ultrasonic testing. The latter technique, mainly based on wave velocity determination, is widely used in the wood industry for mechanical grading of lumber, timber structure inspection, and property assessment of standing trees. Formerly, the wave velocity was determined between two sensors leading to a one-dimensional characterization. More recently, bi-dimensional characterization has been developed using acoustic tomography. The main acoustic tomographs currently available on the market consist of the following: Picus ${ }^{\circledR}$ (Argus Electronic), ARBOTOM ${ }^{\circledR}$ (Rinntech), and ArborSonic (Fakopp). These are the devices used in most published studies (Rust 2000; Gilbert and Smiley 2004; Rabe et al. 2004; Rinn 2004; Divos and Divos 2005; Wang et al. 2007; Deflorio et al. 2008; Li et al. 2012). Other studies 
(Nicolotti et al. 2003; Martinis et al. 2004; Socco et al. 2004) conducted ultrasonic experiments with the PUNDIT device (Portable Ultrasonic Non-destructive Digital Indicating Tester, C.N.S. Electronics, London, UK), operating at $33 \mathrm{kHz}$. The results obtained demonstrated the feasibility of ultrasonic tomography and were confirmed by numerical and experimental studies (Maurer et al. 2006; Schubert 2007; Schubert et al. 2009). Various parameters affect the quality of tomographic images: wavelength, signal-to-noise ratio, number of sensors, and the inversion algorithm used. Signal attenuation correlates closely with the frequency employed, trunk moisture content, orthotropic direction (Beall 2002; Bucur 2006), and the presence of internal decay. In the specific case of ultrasonic tomography, attenuation is also due to the acoustic impedance mismatch between transducers and the wood (Nicolotti et al. 2003; Socco et al. 2004). The received signals are thus mixed with noise and the tomographic images can be biased by unusual propagation time values (low signal-tonoise ratio). In this context, little information has been reported concerning time of flight (TOF) measurements in the acoustic and ultrasonic tomography of wood. TOF in the ultrasonic NDT of wood is usually determined from a userfixed threshold (Kanda et al. 1998; Beall 2002; Bucur 2006; Yanagida et al. 2007). However, statistical techniques such as the Akaike information criterion have also been used as a travel time picker in acoustic tomography (Maurer et al. 2006; Schubert 2007; Schubert et al. 2009). No information was, however, available concerning the accuracy of TOF determination techniques and the comparison between techniques in relation to wood material characterization. The main research questions of this study were thus the following: (1) What is the effect of the signal dynamic on the velocity determination? (2) What is the validity range of the main algorithms considering their systematic and random bias? (3) Is there a difference between a homogeneous material and wood in terms of the velocity determination? Signals used for the TOF computation were analyzed using four different algorithms. One isotropic material and two wood species were tested (parallelepiped samples). The amount of noise added was strictly controlled by conducting the experiments using the conventional experimental protocol for the ultrasonic characterization of materials in a tank.

\section{Material and methods}

\subsection{Experimental protocol}

TOF was determined using four different algorithms and three different samples (1 isotropic material and 2 wood species). Signals were generated with a given signal-to-noise ratio (SNR) ranging from $-3.6 \mathrm{~dB}$ to $+28 \mathrm{~dB}$ (SNR range divided into 20 steps). In all, 100 signals, obtained under the same experimental condition (SNR value), were used to compute TOF values and ultrasonic wave velocities. Mean and standard deviation values were then computed for each experimental condition (20 steps, each with 100 values) to study to what extent the algorithms were able to determine wave velocity.

\subsection{Material}

A sample of polyurethane resin (RP6406) was used as isotropic material (density value $=1,165 \mathrm{~kg} / \mathrm{m}^{3}$, compressional wave velocity $=2,070 \mathrm{~m} / \mathrm{s}$; Peirlinckx et al. 1993). The sample dimensions were $18 \times 110 \times 170 \mathrm{~mm}$ and the ultrasonic beam was collinear to the depth direction $(18 \mathrm{~mm})$. Two species of the same genus (Moraceae) were added to the experiments: Bagassa guianensis Aubl. (Tatajuba, longitudinal modulus= $21,500 \mathrm{MPa}$ and density $=800 \mathrm{~kg} / \mathrm{m}^{3}$ at a moisture content of $12 \%$; source: Tropix database - Cirad, http://tropix.cirad.fr/ en) and Milicia excelsa (Welw.) C.C. Berg (Iroko, longitudinal modulus $=12,800 \mathrm{MPa}$ and density $=640 \mathrm{~kg} / \mathrm{m}^{3}$ at a moisture content of $12 \%$; source: Tropix database - Cirad, http://tropix. cirad.fr/en). The compression wave velocity is mainly governed by the specific modulus. For tropical woody species, this material property is in the range of 6-34 with an average of 17 (measured on 2,864 samples; Tropix database - Cirad). Tatajuba and Iroko were selected for the experiments because their specific modulus was close to the average value for Iroko and close to the maximum value for Tatajuba. The samples were tested in the ultrasonic experiments at moisture content above $30 \%$. The sample dimensions were $10 \mathrm{~mm}(R$ axis $) \times 60 \mathrm{~mm}(T$ axis $) \times 130 \mathrm{~mm}$ ( $L$ axis). The ultrasonic beam was collinear to the $R$ direction.

\subsection{Ultrasonic experiments}

The conventional experimental protocol (Fig. 1) was used to measure ultrasonic velocity in the different materials (Henry

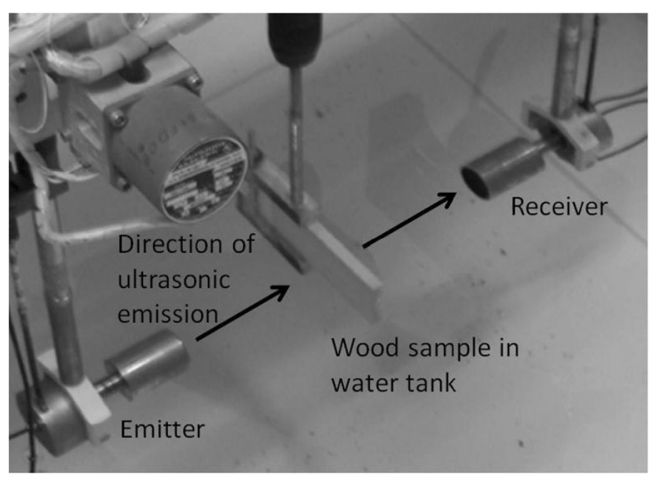

Fig. 1 Experimental setup to measure ultrasonic velocity in transmission mode with normal incidence (wood samples placed in a water tank and tested in the radial direction) 
et al. 2010). Each sample was placed in a tank filled with water. The measurements were taken in transmission (normal incidence) with two transducers set at $500 \mathrm{kHz}$. This frequency was chosen such that the wavelength was shorter than sample thickness. The signal-to-noise ratio (SNR) was varied by changing generator gain. SNR was computed as mean signal power divided by mean noise power. Figure 2 shows two extreme examples of the experimental signals obtained (sampling frequency set at $20 \mathrm{MHz}$ and acquisition duration $51.2 \mu \mathrm{s})$.

Two measurements were taken, one without the sample (reference measurement), the other with the sample. Velocity $C$ in the material was determined by Eq. (1) where $C_{0}$ is the velocity of the ultrasound wave in water, $\Delta t$ is the time difference between the two signals, and $L$ is the thickness of the material.

$$
C=\frac{C_{0}}{1+\frac{C_{0} \Delta t}{L}}
$$

a

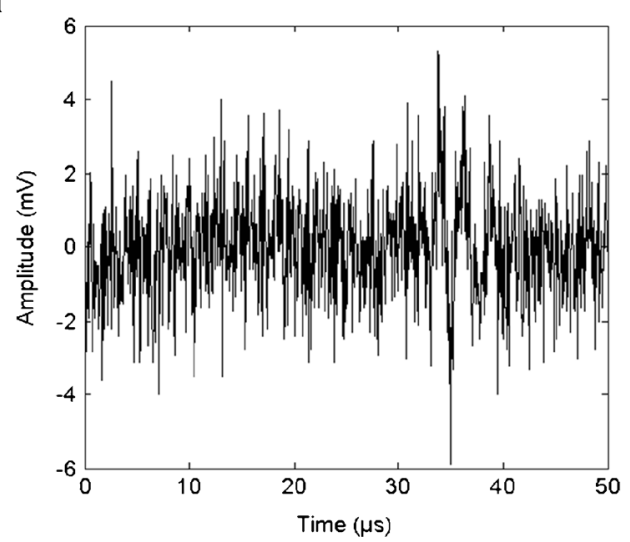

b

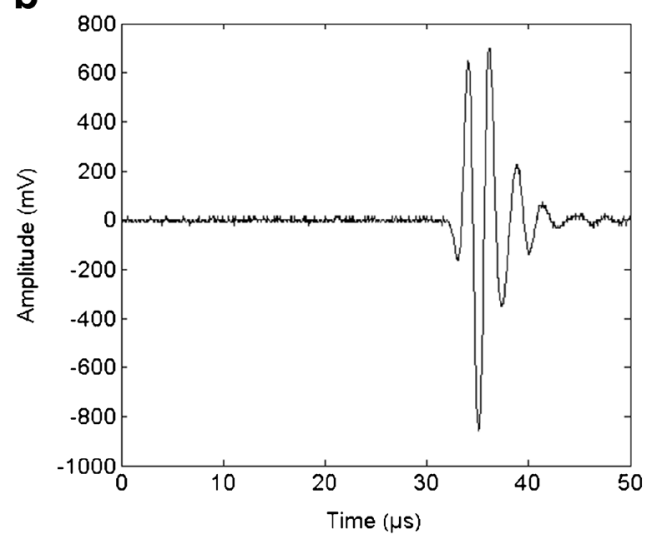

Fig. 2 Experimental signals recorded in transmission mode with a normal incidence obtained with Tatajuba (radial direction). With a very high noise level, $\mathrm{SNR}=-3.6 \mathrm{~dB}$ (a) and a very low noise level, $\mathrm{SNR}=26 \mathrm{~dB}$ (b). Variation of noise level obtained by changing the generator gain

\subsection{Determination of propagation time}

The time difference $\Delta t$ corresponded to the difference between the two first arrival times $\left(\Delta t=t_{1}-t_{0}\right)$ where $t_{0}$ is the TOF for the reference signal and $t_{1}$ is the TOF for the test sample. $t_{0}$ was computed by cross-correlation (reference signal processing method) on the 100 reference signals obtained with the optimal SNR value $(28 \mathrm{~dB})$. The $t_{0}$ value used was the average, which thereafter was considered to be a constant value. The $t_{1}$ values associated with the test samples were determined using the four methods described below:

- The threshold criterion is the method most commonly used in the nondestructive testing of wood. Here, TOF is determined at the point where the instantaneous power of the signal (or its envelope obtained by the Hilbert transform) exceeds the power of the noise (Beall 2002; Bucur 2006). The determination is commonly carried out by (1) a threshold that depends on the statistical properties of the noise or (2) a threshold proportional to the maximum value of the temporal amplitude of the signal (Kanda et al. 1998; Yanagida et al. 2007). In the study reported herein, the threshold was set at five times the standard deviation of the noise.

- The Akaike information criterion (AIC) method has been used since the 1980s in geophysics. Based on the assumption that a time series (e.g., discrete signals) can be divided into locally stationary segments (each modeled as an autoregressive process), the AIC is used to determine both the order of the autoregressive models and the TOF of the longitudinal and transverse waves (Sleeman and van Eck 1999). The point at which the AIC is minimized determines the optimal separation between the two time series (noise and signal). The AIC method has already been reported by Maurer et al. (2006) and Schubert (2007 and 2009). TOF is determined by computing the AIC function using Eq. (2) (Maeda 1985; Zhang et al. 2003), where $x$ [k] is the signal at index $k, N$ is the length of the signal, and var is the variance.

$$
\begin{aligned}
A I C[k]= & k \cdot \log (\operatorname{var}(x[1, \ldots, k])) \\
& +(N-k) \cdot \log (\operatorname{var}(x[k+1, \ldots, N]))
\end{aligned}
$$

- The Hinkley criterion is a TOF determination method that has been used in the nondestructive testing of concrete (Hinkley 1971; Grosse and Reinhardt 1999; Kurz et al. 2005). It is based on the modified partial energy of the signal (Eq. 3). A factor $\alpha$ is introduced to adjust the global minimum position of the modified partial energy of the signal (Kurz et al. 2005). The value chosen for $\alpha$ significantly affects the results and adaptive selection can be 
used to avoid dispersion in a group of similar propagation times. For $\alpha=1$, the Hinkley criterion is reduced to a comparison between the instantaneous power of the signal at point $k$, and the mean power of the signal. TOF is therefore associated with the index $k$ (instant at which instantaneous power is greater than the mean power of the signal).

$$
S[k]=\sum_{p=1}^{k}\left(x_{p}^{2}\right)-\frac{k}{\alpha \cdot N} \sum_{p=1}^{N}\left(x_{p}^{2}\right)
$$

- The short-term Fisher variance criterion was developed for this study (two tailed $F$ test). This criterion is therefore based on a comparison of the variance over two continuous segments of the same size (Eq. 4). In our case, size $n$ was set at 30 and the risk level at 1/1,000.

$$
F[k]=\frac{\operatorname{var}(x[k, \ldots, k+n-1])}{\operatorname{var}(x[k+n, \ldots, k+2 \cdot n-1])}
$$

\section{Results}

Figure 3 shows velocities computed from average propagation times for each SNR level. The SNR ranges were slightly different for the various materials tested because of differences in signal attenuation (given that resin attenuates less than wood, the minimum SNR for resin was found to be higher than that for wood). The trends illustrated in Fig. 3 were nevertheless similar for resin, Tatajuba, and Iroko. When the SNR reached $28 \mathrm{~dB}$, the velocity values obtained by the different methods corresponded to about $2,100 \pm 150 \mathrm{~m} / \mathrm{s}$ for resin, $1,600 \pm 150 \mathrm{~m} / \mathrm{s}$ for Tatajuba, and $1,400 \pm 150 \mathrm{~m} / \mathrm{s}$ for Iroko. The variation $( \pm 150 \mathrm{~m} / \mathrm{s})$ was virtually identical for all the test materials. Also, the different methods consistently yielded results in the same order, and this for all the materials: Fisher gave the highest velocity value, followed by Threshold, AIC, then Hinkley. AIC was the most sensitive to noise level (substantial velocity overestimation for SNR values below $5 \mathrm{~dB}$ ). Average velocity decreased as SNR increased to $10 \mathrm{~dB}$. It then reached a minimum before gradually increasing to a limit value at $28 \mathrm{~dB}$. Hinkley showed a similar trend to AIC, but variations were less pronounced as the curve was virtually flat after approximately $5 \mathrm{~dB}$. The results of the Fisher and Threshold methods tended to increase with SNR. The two curves were very similar but Fisher showed the greatest variation range $(300 \mathrm{~m} / \mathrm{s}$ in the SNR range).

Figure 4 shows the velocity variation range for each SNR level. This variation range was computed from the standard deviation of the propagation times. It corresponded to the
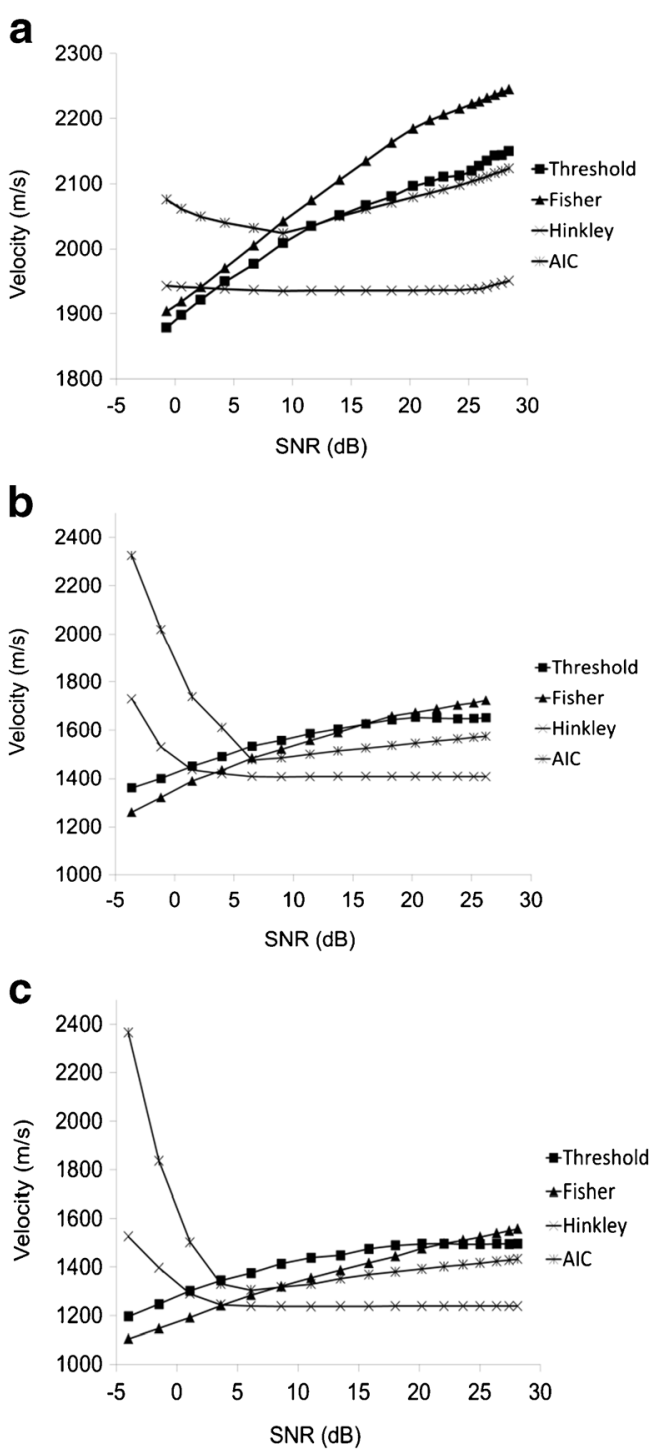

Fig. 3 Velocity computed from average propagation times for each noise level (SNR). Average on 100 determinations of propagation times according to four signal processing techniques (Threshold, Fisher, Hinkley, AIC). a Resin, b Tatajuba, c Iroko

difference between velocity at time $t_{1}$, plus a standard deviation, and velocity at time $t_{1}$, minus a standard deviation. In what follows, this velocity variation range is referred to as the measurement error. This measurement error decreased as SNR increased, and this for all the methods used. Measurement error was found to be higher for the wood samples than for the resin sample (for example, average measurement error for the Threshold method was $400 \mathrm{~m} / \mathrm{s}$ for resin and $800 \mathrm{~m} / \mathrm{s}$ for wood). The methods could once again be arranged in the same order, and this once again for all the test materials $(\mathrm{SNR}=$ $28 \mathrm{~dB}$ ): Threshold had the highest error followed by Fisher, AIC, then Hinkley. Marked differences were also noted between the methods. Measurement error for the AIC and Hinkley methods was greatly impacted by noise level when SNR was below $10 \mathrm{~dB}$. It exceeded $1,200 \mathrm{~m} / \mathrm{s}$ in Tatajuba, 

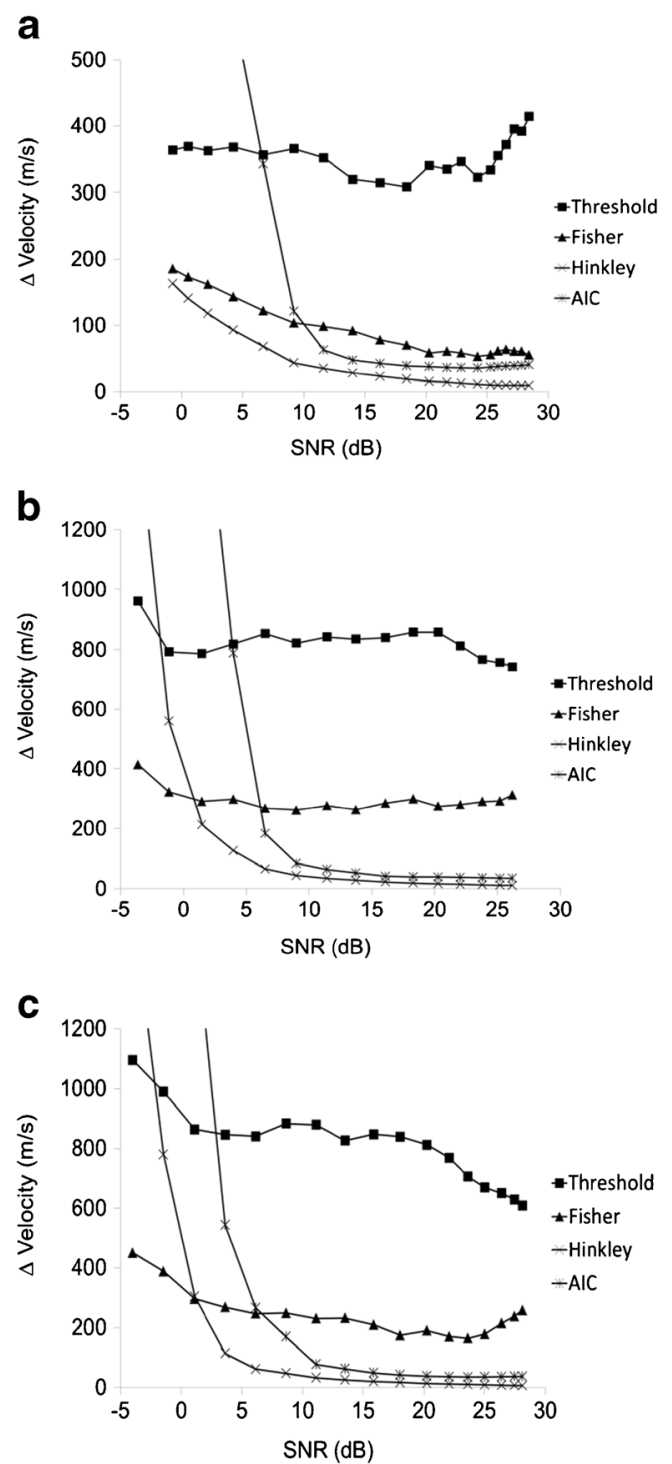

Fig. 4 Velocity variation range computed from the standard deviation of propagation times for each noise level (SNR). Standard deviation on 100 determinations of propagation times according to four signal processing techniques (Threshold, Fisher, Hinkley, AIC). Variation range defined as the difference between velocity at the average propagation time plus a standard deviation and velocity at the average propagation time minus a standard deviation. a Resin, b Tatajuba, c Iroko

associated for example $(\mathrm{SNR}=0 \mathrm{~dB})$ with an average velocity of $1,500 \mathrm{~m} / \mathrm{s}$ (Hinkley) and 2,000 m/s (AIC). In this case, the relative error then exceeded approximately $40 \%$. Conversely, when SNR exceeded $10 \mathrm{~dB}$, these two methods gave the lowest measurement error (less than $50 \mathrm{~m} / \mathrm{s}$ at $26 \mathrm{~dB}$ for Tatajuba, with a relative error of less than $2 \%$ ). The Fisher method had a relatively low measurement error that decreased with the SNR (between 200 and $50 \mathrm{~m} / \mathrm{s}$ for resin, between 400 and $200 \mathrm{~m} / \mathrm{s}$ for wood). The worst performance was seen with the Threshold method: similar trend to the Fisher method but with twice the error levels.

\section{Discussion}

The average velocity value of $2,100 \mathrm{~m} / \mathrm{s}$ obtained for the resin (average for all signal processing methods at a SNR of $28 \mathrm{~dB}$ ) was found to be consistent with manufacturer data (resin manufactured by CNRS-LMA, Peirlinckx et al. 1993). Specific moduli computed for Tatajuba and Iroko were 27 and 20, respectively (Cirad database). It was deduced from these values that the transverse velocity of Tatajuba was greater than that of Iroko. This hypothesis was confirmed experimentally with an average velocity of $1,600 \mathrm{~m} / \mathrm{s}$ for Tatajuba and $1,400 \mathrm{~m} / \mathrm{s}$ for Iroko (average for all signal processing methods at a SNR of 26 and $28 \mathrm{~dB}$, respectively). The ultrasonic wave propagation was collinear to the radial direction of the wood samples. This propagation direction was chosen because ultrasonic tomography is done in the transverse cross-section of trees. The wood samples were cut in the material axes (radial, tangential, and longitudinal). However, due to annual ring curvature, wood mechanical property gradients exist in the tested direction and, in addition to experimental reproducibility errors, could explain some of the result dispersions.

The trends depicted in Fig. 3 are explained by the diagram in Fig. 5. The explanation given below was true for Hinkley and AIC when SNR was greater than $10 \mathrm{~dB}$. The demarcation between the left part (noise) and the right part (deterministic signal) shifted toward positive times as the noise level increased. Therefore, propagation time tends to increase as SNR decreases, especially when the frequency of the higher energy wave is low (curvature at the start of the signal). This phenomenon will be pronounced for waves generated by hitting the trunk with a hammer since the highest energy wave corresponds to the first resonance frequency of the trunk (a few kilohertz). Computed velocity in our study was seen to decrease as propagation time increased, as observed in Fig. 3 (velocity decreased as SNR decreased).

The Threshold method is governed by the multiplier parameter of the standard deviation. If this parameter is low, systematic bias will also be low, but random bias will be very high because the probability of belonging to the noise area will be high. Our experimental measurements not only in the laboratory on small samples (in a tank or in contact) but also in

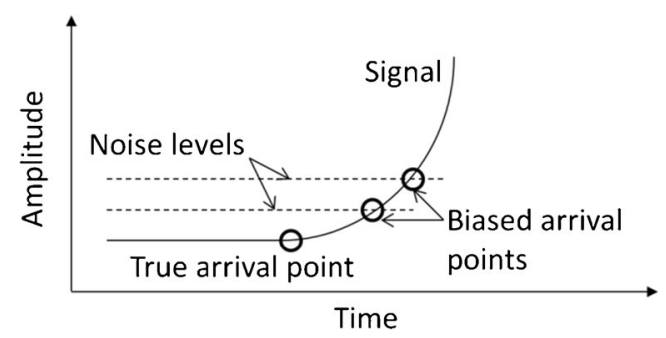

Fig. 5 Schematic drawing of the effect of noise level on the determination of the first arrival point of the traveling waves (propagation time) by the signal processing techniques 
tomography helped us determine an acceptable compromise by setting this parameter to 5 (i.e., the value set in this study). The Fisher method was found to be well suited when SNR is low. It continued to give accurate values even when the signal was almost drowned in the noise (Fig. 2). This method is governed by the size of the segments for analysis. The segment must be smaller than the lifetime of the signal (the deterministic part of the signal). Segment size in our study was set at 30 points for an acceptable estimate of the variance. The disadvantage here is that the size of a segment delocalizes the arrival point in time: this method determines whether two consecutive segments are different, but the arrival point can be anywhere in the second segment. It was set here in the middle of the second segment. The trends associated with the Hinkley and AIC methods (Figs. 3 and 4) showed that these algorithms gave inaccurate velocity values when SNR was very low (SNR $<10 \mathrm{~dB}$ ). This has already been reported by Zhang et al. (2003). If the SNR is too low, the power of the signal is equal to the power of the noise and it is thus impossible to determine the arrival point with a Hinkley criterion. The same remark can be made for the AIC method where the variance of the left part of the signal is successively compared with the variance of the right part. If signal lifetime is short compared to the total duration of the acquisition, and if the noise level is high, the variances are equal and it is impossible to determine the arrival point.

The results presented in Figs. 3 and 4 are associated with the systematic error (Fig. 3) and the random error (Fig. 4), respectively. The systematic bias is the difference between the true value and the average of the experimental values. In our study, the true value was unknown and we thus chose to focus on the average of the experimental values. The random error is the dispersion of the experimental values around the average of these values. We chose in our study to use the width of the confidence interval with 1 standard deviation rather than the more conventionally computed half-interval. In the tomography of standing trees, the SNR variation depends not only on the distance between probes but also on the presence of defects. The computed velocities follow the trends presented in Figs. 3 and 4. In the particular case of a variable SNR, the appropriate method is therefore that which provides the most constant average velocity (Fig. 3) and the lowest possible measurement error (Fig. 4). The Hinkley method appears to be that most suited when the SNR exceeds $10 \mathrm{~dB}$. The Fisher method is suitable for very noisy signals (minimum random error) but systematic bias here is not constant. It would be useful to combine these two methods into a single algorithm: Fisher to identify a specific part of the signal; Hinkley applied to this part to identify arrival time point more precisely. Another method of investigation would be to use the Chirp analysis (based on the correlation methods between a reference Chirp signal and the experimental output signal; Gan et al. 2005), but this should be used directly under field conditions (tree tomography) in order to take account of the distortion of this specific wave shape due to attenuation and dispersion.

\section{Conclusion}

Ultrasonic velocities of one homogeneous material and two wood species were computed according to signal-to-noise ratio (SNR) in order to determine the most accurate signal processing technique. The experimental protocol was designed (1) to study the effect of the signal dynamic on the velocity computation, (2) to determine the validity range of the signal processing techniques, and (3) to observe the difference between the tested materials.

It was demonstrated from the experiments that the velocity values increased with the SNR level, i.e., when the signal dynamic increased (this was particularly true for the Fisher and Threshold methods). This phenomenon was due to the shift of the wave arrival detection toward the positive times when the noise level increased.

The Akaike and Hinkley methods were strongly influenced by the SNR level below $5 \mathrm{~dB}$ for the velocity value and below $10 \mathrm{~dB}$ for the determination error. The validity range of these methods was thus between 10 and $30 \mathrm{~dB}$. The Hinkley method had the lowest determination error. The Fisher and Threshold methods tended to a quasi-constant velocity value between 20 and $30 \mathrm{~dB}$. The determination error of the Fisher method was, however, lower by a factor two compared with the Threshold method. Furthermore, it was noticed that the error of the Fisher method remained low and stable for a SNR below $10 \mathrm{~dB}$.

No difference was found between the resin and the wood samples concerning the variation of the computed velocity according to the noise level. However, the determination error was found to be higher for the wood samples than for the resin. The signal attenuation was also higher for the wood samples.

The authors recommend using the Fisher method when the SNR is below $10 \mathrm{~dB}$ (strong wave attenuation). The Hinkley method should be used in the case of a SNR above $10 \mathrm{~dB}$. A combination of these two methods could also be employed regardless of the noise level.

\section{References}

Beall F (2002) Overview of the use of ultrasonic technologies in research on wood properties. Wood Sci Technol 36:197-212

Bucur V (2003) Non-destructive Characterization and Imaging of Wood. Springer Series in Wood Science 
Bucur V (2006) Acoustics of wood. Springer Series in Wood Science, 2nd edition

Deflorio G, Fink S, Schwarze FWMR (2008) Detection of incipient decay in tree stems with sonic tomography after wounding and fungal inoculation. Wood Sci Technol 42:117-132

Divos F, Divos P (2005) Resolution of stress wave based acoustic tomography. 14th international symposium on non-destructive testing of wood, Germany 309-314

Gan TH, Hutchins DA, Green RJ, Andrews MK, Harris PD (2005) Noncontact, high-resolution ultrasonic imaging of wood samples using coded chirp waveforms. IEEE Trans UFFC 52:280-288

Gilbert EA, Smiley ET (2004) Picus sonic tomography for the quantification of decay in white oak (Quercus alba) and hickory (Carya spp.). J Arb 30:277-281

Grosse C, Reinhardt HW (1999) Schallemissionsquellen automatisch lokalisieren: Entwicklung eines algorithmus. Materialprüfung 41: 342-347

Henry M, Ayrault C, Castagnède B, El Abidine FZ, Depollier C, Fellah M, Lauriks W, Sahraoui S (2010) Metrology of physical parameters of absorbing materials. In: Bruneau M, Potel C (eds) Materials and acoustics handbook. ISTE, London

Hinkley D (1971) Inference about the change-point from cumulative sum tests. Biometrika 58:509-523

Kanda S, Shioya K, Yanagiya Y, Tamura Y, Adachi K (1998) Ultrasonic TOF-CT system for wooden pillars. Ultrason Symp IEEE 1:743746

Kurz JH, Grosse CU, Reinhardt HW (2005) Strategies for reliable automatic onset time picking of acoustic emissions and of ultrasound signals in concrete. Ultrasonics 43:538-546

Li L, Wang X, Wang L, Allison R (2012) Acoustic tomography in relation to $2 \mathrm{D}$ ultrasonic velocity and hardness mappings. Wood Sci Technol 46:551-561

Maeda N (1985) A method for reading and checking phase times in autoprocessing system of seismic wave data. J Seismol Soc Jpn 38:365-379

Martinis R, Socco LV, Sambuelli L, Nicolotti G, Schmitt O, Bucur V (2004) Tomographie ultrasonore pour les arbres sur pied. Ann For Sci 61(2):157-162

Maurer H, Schubert S, Bächle F, Clauss S, Gsell D, Dual J, Niemz P (2006) A simple anisotropy correction procedure for acoustic wood tomography. Holzforschung 60:567-573
Nicolotti G, Socco LV, Martinis R, Godio A, Sambuelli L (2003) Application and comparison of three tomographic techniques for detection of decay in trees. J Arb 29:66-78

Peirlinckx L, Pintelon R, Van Biesen L (1993) Identification of parametric models for ultrasonic wave propagation in the presence of absorption and dispersion. IEEE Trans Ultrason Ferroelec Freq Contr 40:302-312

Pellerin R, Ross R (2002) Non-destructive evaluation of wood. Forest Products Society, Madison

Rabe C, Ferner D, Fink S, Schwarze FWMR (2004) Detection of decay in trees with stress waves and interpretation of acoustic tomograms. J Arb 28:3-19

Rinn F (2004) Holzanatomische grundlagen der schall-tomographie an baumen. Neue Landschaft 7:44-47

Rust S (2000) A new tomographic device for the non-destructive testing of trees. Proceedings of the 12th international symposium on nondestructive testing of wood, Sopron, Hungary, University of West Hungary 233-237

Schubert S (2007) Acousto-ultrasound assessment of inner wooddecay in standing trees: possibilities and limitations. $\mathrm{PhD}$ thesis, ETH, Zurich

Schubert S, Gsell D, Dual J, Motavalli M, Niemz P (2009) Acoustic wood tomography on trees and the challenge of wood heterogeneity. Holzforschung 63:107-112

Sleeman R, van Eck T (1999) Robust automatic P-phase picking: an online implementation in the analysis of broadband seismogram recordings. Phys Earth Planet 113:265-275

Socco LV, Sambuelli L, Martinis R, Comino E, Nicolotti G (2004) Feasibility of ultrasonic tomography for non-destructive testing of decay on living trees. Research in Non-destructive Evaluation 15(1): $31-54$

Wang X, Allison RB, Wang L, Ross RJ (2007) Acoustic tomography for decay detection in red oak trees. Research Paper FPL-RP-642, U.S. Department of Agriculture, Forest Service, Forest Products Laboratory, Madison, WI

Yanagida H, Tamura Y, Kim KM, Lee JJ (2007) Development of ultrasonic time-of-flight computed tomography for hard wood with anisotropic acoustic property. Jpn J Appl Phys 46:53215325

Zhang H, Thurber C, Rowe C (2003) Automatic p-wave arrival detection and picking with multiscale wavelet analysis for single-component recordings. Bull Seism Soc Amer 93:1904-1912 